\title{
Moments of meson distribution functions with dynamical twisted mass fermions
}

\author{
Rémi Baron \\ SPhN-DAPNIA, CEA Saclay, 91191 Gif sur Yvette, France \\ E-mail: remi.baronecea.fr
}

\section{Stefano Capitani}

Fakultät für Physik, Universität Bielefeld, Universitätsstrasse, 33615 Bielefeld, Germany

E-mail: capitani@physik.uni-bielefeld.de

\section{Jaume Carbonell}

Laboratoire de Physique Subatomique et Cosmologie, 53 Av des martyrs, 38026 Grenoble, France

E-mail: carbonelelpsc.in2p3.fr

\section{Karl Jansen}

DESY, Platanenallee 6, 15738 Zeuthen, Germany

E-mail: Karl.Jansen@desy.de

\section{Zhaofeng Liu* and Olivier Pène}

Laboratoire de Physique Théorique (Bât. 210), Université de Paris XI, Centre d'Orsay, 91405

Orsay-Cedex, France

E-mail: zhaofeng.liudth.u-psud.fr, Olivier.Penedth.u-psud.fr

\section{Carsten Urbach}

Theoretical Physics Division, Dept. of Mathematical Sciences, University of Liverpool,

Liverpool L69 7ZL, UK

E-mail: Carsten.Urbachaliverpool.ac.uk

\section{On behalf of the ETM Collaboration}

We present our preliminary results on the lowest moment $\langle x\rangle$ of quark distribution functions of the pion using two flavor dynamical simulations with Wilson twisted mass fermions at maximal twist. The calculation is done in a range of pion masses from 300 to $500 \mathrm{MeV}$. A stochastic source method is used to reduce inversions in calculating propagators. Finite volume effects at the lowest quark mass are examined by using two different lattice volumes. Our results show that we achieve statistical errors of only a few percent. We plan to compute renormalization constants non-perturbatively and extend the calculation to two more lattice spacings and to the nucleons.

The XXV International Symposium on Lattice Field Theory

July 30 - August 42007

Regensburg, Germany

\footnotetext{
* Speaker.
} 


\section{Introduction}

Deep inelastic structure functions of mesons and nucleons are interesting since those functions give us information about the momentum and spin carried by quarks and gluons inside hadrons. Lattice QCD can calculate moments of structure functions from first principles. By doing an operator product expansion on the hadronic tensor, the moments of structure functions are related to reduced matrix elements of certain local operators. For example, for the case of scattering of a spin one particle, the details are given in Ref.[1]. On the lattice, calculations on moments of structure functions of mesons and nucleons can be found in, e.g., Ref.[2, 3, 4, 5].

The Wilson twisted mass formulation of lattice QCD was introduced in Ref.[6, 7]. It provides automatic $\mathscr{O}(a)$ improvement when tuned to maximal twist, which can be achieved by setting the PCAC mass to zero. Also, twisted mass fermions are protected from unphysical fermion zero modes, thus the problem of exceptional configurations is avoided. Quenched simulations with Wilson twisted mass fermions have been shown to be successful[ $8,9,10,11]$. Specifically, a quenched calculation of the lowest moment of the quark distribution function $\langle x\rangle$ in a pion was done in Ref.[12]. Recently, two flavor dynamical simulations[13] with Wilson twisted mass fermions gave precise results on low energy constants in the chiral effective Lagrangian. Using those configurations, in this work we calculate $\langle x\rangle$ in a pion. The computation is performed at pion masses in the range of $\sim 300-500 \mathrm{MeV}$. In Table 1, the parameters of our simulations are collected. The gauge action used in the simulations is the tree-level Symanzik improved gauge action. For more details of our data, please see Ref.[14].

\begin{tabular}{ccccccc}
\hline \hline$\beta$ & $a(\mathrm{fm})$ & $a \mu$ & $m_{\pi}(\mathrm{GeV})$ & $L^{3} \times T$ & $N_{\text {meas }}$ & $\langle x\rangle^{\text {bare }}$ \\
\hline 3.9 & $0.0855(6)$ & 0.0100 & $0.4839(12)$ & $24^{3} \times 48$ & 170 & $0.295(3)$ \\
& & 0.0085 & $0.4470(12)$ & $24^{3} \times 48$ & 167 & $0.279(4)$ \\
& & 0.0064 & $0.3903(9)$ & $24^{3} \times 48$ & 250 & $0.279(5)$ \\
& & 0.0040 & $0.3131(16)$ & $24^{3} \times 48$ & 230 & $0.268(8)$ \\
& & 0.0040 & $0.3082(55)$ & $32^{3} \times 64$ & 316 & $0.251(8)$ \\
\hline \hline
\end{tabular}

Table 1: Simulation parameters, the number of measurements $\left(N_{\text {meas }}\right)$ and preliminary results of $\langle x\rangle$ bare . Measurements were done on gauge configurations separated by $20 \mathrm{HMC}$ trajectories with trajectory length $\tau=1 / 2$.

\section{Methodology}

By using the optical theorem, the cross section of deep inelastic scatterings can be related to the hadronic tensor or the imaginary part of the forward current-hadron scattering amplitude $W^{\mu \nu}$, which is given by

$$
W^{\mu v}\left(p, q, \lambda, \lambda^{\prime}\right)=\frac{1}{4 \pi} \int d^{4} x e^{i q \cdot x}\left\langle p, \lambda^{\prime}\left|\left[j^{\mu}(x), j^{v}(0)\right]\right| p, \lambda\right\rangle .
$$

Here $\lambda$ and $\lambda^{\prime}$ are the polarization of the target hadron. $p$ and $q$ are the momenta of the hadron and the virtual photon. Depending on the spin of the hadron, the above tensor $W^{\mu v}$ can be decomposed 
into a number of independent structure functions. The spin-averaged structure functions $F_{1}\left(x, Q^{2}\right)$ and $F_{2}\left(x, Q^{2}\right)$ can tell us overall densities of quarks and gluons in a hadron. In the parton model, to leading order, the single flavor structure function $F_{1}(x)$ is half the probability of finding a quark with momentum fraction $x$. If defining the $n$th moment of a function $f(x)$ as

$$
M_{n}(f)=\int_{0}^{1} x^{n-1} f(x) d x,
$$

then for the pion, to leading twist order, we have from the operator product expansion of $W^{\mu v}$

$$
2 M_{n}\left(F_{1}\right)=C_{n}^{(1)} v_{n}, \quad M_{n-1}\left(F_{2}\right)=C_{n}^{(2)} v_{n}, \quad(n \geq 2, \text { even }),
$$

where $C_{n}^{(k)}=1+\mathscr{O}\left(\alpha_{s}\right)$ are the Wilson coefficients, and the reduced matrix element $v_{n}$ is defined by

$$
\left\langle\vec{p}\left|\mathscr{O}^{\left\{\mu_{1} \cdots \mu_{n}\right\}}-\operatorname{traces}\right| \vec{p}\right\rangle=2 v_{n}\left[p^{\mu_{1}} \cdots p^{\mu_{n}}-\text { traces }\right] .
$$

Here $\{\cdots\}$ means symmetrization on the Lorentz indices and the twist- 2 operators are given by

$$
\mathscr{O}^{\mu_{1} \cdots \mu_{n}}=\left(\frac{i}{2}\right)^{n-1} G_{f f^{\prime}} \bar{\psi}_{f} \gamma^{\mu_{1}} \stackrel{\leftrightarrow}{D} \mu_{2} \cdots \stackrel{\leftrightarrow}{D}^{\mu_{n}} \psi_{f^{\prime}}
$$

where $\stackrel{\leftrightarrow}{D}=\vec{D}-\overleftrightarrow{D}$ and $G_{f f^{\prime}}$ is a diagonal flavor matrix.

To compute the lowest moment of the quark distribution function $\langle x\rangle$, we need to consider $n=2$ in Eq.(2.3). The corresponding twist- 2 operator has two irreducible representations on the lattice[15]. In our calculation we use the following operator

$$
\mathscr{O}_{44}(x)=\frac{1}{2} \bar{u}(x)\left[\gamma_{4} \stackrel{\leftrightarrow}{D}_{4}-\frac{1}{3} \sum_{k=1}^{3} \gamma_{k} \stackrel{\leftrightarrow}{D}_{k}\right] u(x)
$$

where $D_{\mu}=\frac{1}{2}\left(\nabla_{\mu}+\nabla_{\mu}^{*}\right)$ with $\nabla \mu\left(\nabla_{\mu}^{*}\right)$ being the usual forward (backward) derivative on the lattice. With the above operator, no external momentum is needed in our calculation, which is advantageous since an external momentum increases the noise to signal ratio. One can also use an operator from the other representation, which needs an external momentum. In the continuum limit, the two operators should give the same result on $\langle x\rangle$.

From Eq.(2.4) the bare moment $\langle x\rangle$ bare is given by

$$
\langle x\rangle^{\text {bare }}=v_{2}=\frac{1}{2 m_{\pi}^{2}}\left\langle\pi, \overrightarrow{0}\left|\mathscr{O}_{44}\right| \pi, \overrightarrow{0}\right\rangle,
$$

where the matrix element $\left\langle\pi, \overrightarrow{0}\left|\mathscr{O}_{44}\right| \pi, \overrightarrow{0}\right\rangle$ between two pions at rest is calculated from the ratio of the following 3-point function and 2-point function with a source at $t=0$ and a sink at $T / 2$ :

$$
\left\langle\pi, \overrightarrow{0}\left|\mathscr{O}_{44}\right| \pi, \overrightarrow{0}\right\rangle=4 m_{\pi} \frac{C_{44}(t)}{C_{\pi}(T / 2)} \quad(0 \ll t \ll T / 2) .
$$

Here

$$
\begin{gathered}
C_{44}(t)=\sum_{\vec{x} \vec{y}}\left\langle P S(T / 2, \vec{x}) \mathscr{O}_{44}(t, \vec{y}) P S^{\dagger}(0,0)\right\rangle, \\
C_{\pi}(T / 2)=\sum_{\vec{x}}\left\langle P S(T / 2, \vec{x}) P S^{\dagger}(0,0)\right\rangle,
\end{gathered}
$$


and $P S(x)=\bar{u}(x) \gamma_{5} d(x)$ is the interpolating field for the pion. There are two contributions in the Wick contractions of $C_{44}(t)$ : one connected diagram and one disconnected diagram. The disconnected contribution is ignored in our calculation at this moment, but will be computed by using a stochastic source method.

The above two and three point correlators are evaluated by using a stochastic time slice source (Z(2)-noise in both real and imaginary part) $[16,17,18]$ for all color, spin and spatial indices. i.e., the quark propagator $X_{\beta}^{b}(y)$ is obtained by solving

$$
\sum_{y} D_{\alpha \beta}^{a b}(z, y) X_{\beta}^{b}(y)=\xi(\vec{z})_{\alpha}^{a} \delta_{z_{0}, 0} \quad(\text { source at } t=0),
$$

where the $\mathrm{Z}(2)$ random source $\xi(\vec{z})_{\alpha}^{a}$ satisfies the random average condition

$$
\left\langle\xi^{*}(\vec{x})_{\alpha}^{a} \xi(\vec{y})_{\beta}^{b}\right\rangle=\delta_{\vec{x}, \vec{y}} \delta_{a, b} \delta_{\alpha, \beta} .
$$

The generalized propagator[19] $\Sigma_{\beta}^{b}(y)$ needed in computing $C_{44}(t)$ is obtained by solving

$$
\sum_{y} D_{\alpha \beta}^{a b}(z, y) \Sigma_{\beta}^{b}(y)=\gamma_{5} X_{\alpha}^{a}(z) \delta_{z_{0}, T / 2} \quad(\text { sink at } t=T / 2) .
$$

The advantage of using the above stochastic source compared with a point source is that much less inversions are needed. With a point source, 24 inversions per gauge configuration are needed: 12 ( 3 colors $\times 4$ spins) for the quark propagator and 12 for the generalized propagator. With the stochastic source, only two inversions are needed. One for the quark propagator, another one for the generalized propagator. Note that this saving ratio in the number of inversions is special for the pseudoscalar meson. For other meson correlators, smaller saving ratios can be achieved.

In our data analysis, measurements are done on gauge configurations separated by $20 \mathrm{HMC}$ trajectories with trajectory length $\tau=1 / 2$. Statistical errors are from a Gamma-function analysis[20].

\section{Preliminary results and outlook}

At the smallest quark mass, $a \mu=0.0040$, we calculated $\langle x\rangle$ using both a point source method and the above stochastic source method on the $24^{3} \times 48$ lattice. The results are in agreement within errors. Fig. 1 shows the comparison of the pion effective masses obtained from the two methods on 230 gauge configurations. As we can see, the statistical errors from the two methods are of the same size. In Fig. 2, we compare the results of $\langle x\rangle$ bare obtained by using the two sources. The two plateaus of $\langle x\rangle$ bare around $T / 4$ and $3 T / 4$ are averaged to increase statistics. Again, we find the statistical errors from the two methods are comparable. However, the stochastic source method is much cheaper in computer time.

Our preliminary results of $\langle x\rangle^{\text {bare }}$ are collected in the last column of Table 1. We only give bare quantities here since the renormalization constant of the matrix element has not been calculated yet, which we plan to compute non-perturbatively. Fig. 3 shows the results of $\langle x\rangle$ bare against the pion mass squared. The cross in the graph at the lowest quark mass is from the $32^{3} \times 64$ lattice. It shows that the finite lattice volume effects are not big. A linear extrapolation in $m_{\pi}^{2}$ gives $\langle x\rangle^{\text {bare }}=0.246(10)$ in the chiral limit (using only the points from the $24^{3} \times 48$ lattice). 


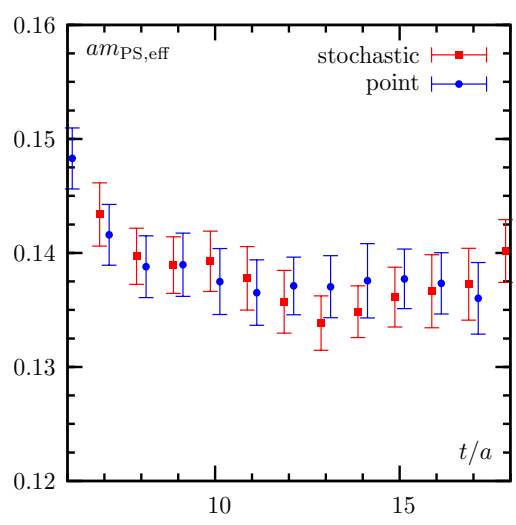

Figure 1: Comparison of the pion effective masses for $a \mu=0.004$ obtained from a point source and a stochastic source on the $24^{3} \times 48$ lattice. They are from the same 230 gauge configurations.
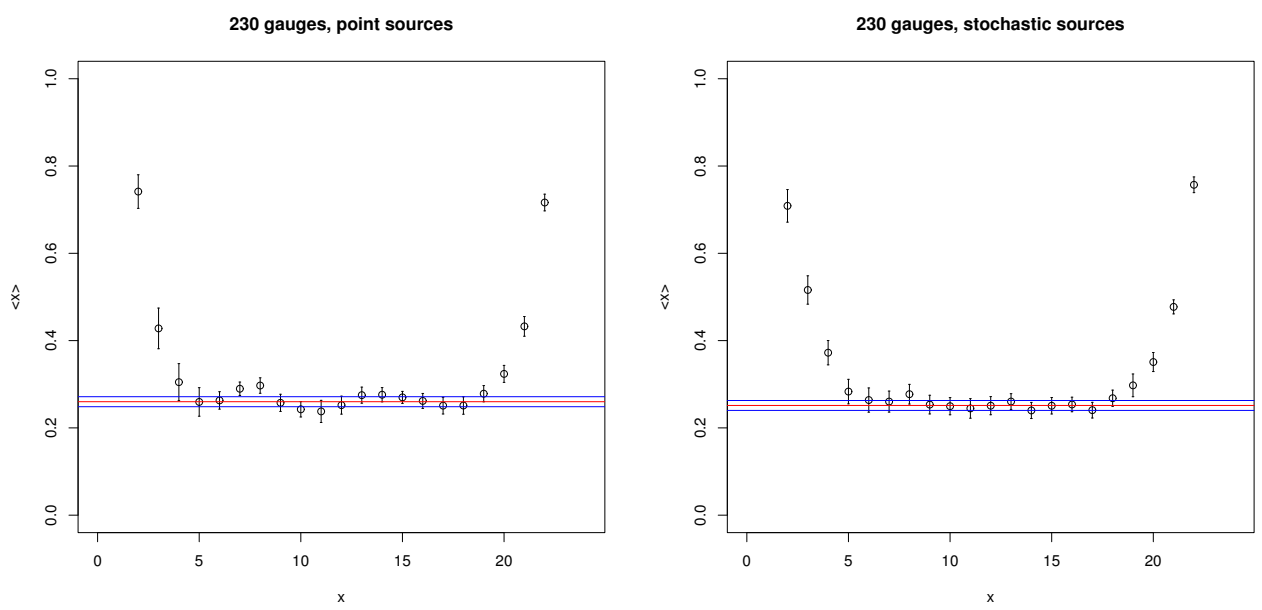

Figure 2: $\langle x\rangle^{\text {bare }}$ for $a \mu=0.004$ obtained from a point source and a stochastic source. We show the average of the two plateaux around $T / 4$ and $3 T / 4$. The stochastic source method is much cheaper in computer time.

Using Wilson twisted mass fermions, our two flavor dynamical simulations go down to a pion mass of around $300 \mathrm{MeV}$. Our first attempt in this work shows we can get $\langle x\rangle$ with small statistical errors of a few percent. This will enable us to compare numerical results of $\langle x\rangle$ in pions and nucleons with predictions from the chiral perturbation theory (see for example [21, 22, 23]). With simulations at two more lattice spacings, we will be able to do an extrapolation to the continuum limit (the data analysis is in progress). For the future, we plan to compute the disconnected diagrams using a stochastic source method and calculate the renormalization constant non-perturbatively. We also plan to compute $\langle x\rangle$ in nucleons.

\section{Acknowledgments}

The numerical calculations were performed on the ZOO cluster at LPT in Orsay, the computers 


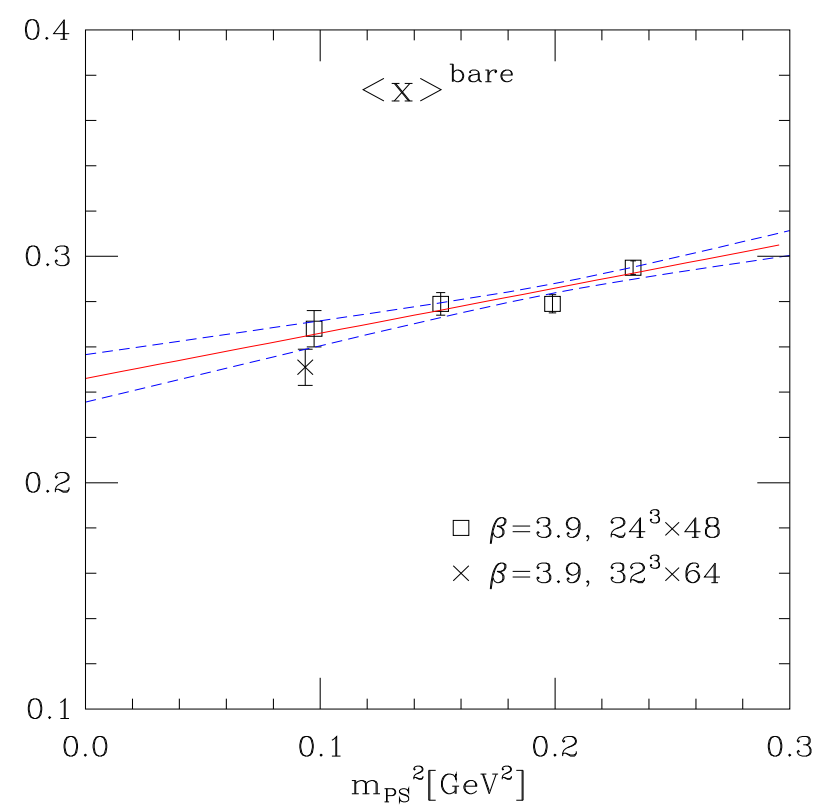

Figure 3: $\langle x\rangle^{\text {bare }}$ against the pion mass squared. Finite size effects at the lowest pion mass are not big. A linear extrapolation gives $\langle x\rangle^{\text {bare }}=0.246(10)$ in the chiral limit (using only the points from the $24^{3} \times 48$ lattice).

of the CCRT (Bruyère-le-Chatel) computing center and the Blue Gene/L in Jülich. We thank Chris Michael for discussions on the stochastic source method. Zhaofeng Liu thanks Stefan Schaefer and Andrea Shindler for valuable discussions.

\section{References}

[1] P. Hoodbhoy, R. L. Jaffe and A. Manohar, Nucl. Phys. B 312 (1989) 571.

[2] M. Göckeler, R. Horsley, E. M. Ilgenfritz, H. Perlt, P. Rakow, G. Schierholz and A. Schiller, Phys. Rev. D 53 (1996) 2317 [arXiv:hep-lat/9508004].

[3] C. Best et al., Phys. Rev. D 56 (1997) 2743 [arXiv:hep-lat/9703014].

[4] M. Guagnelli, K. Jansen, F. Palombi, R. Petronzio, A. Shindler and I. Wetzorke [Zeuthen-Rome (ZeRo) Collaboration], Eur. Phys. J. C 40 (2005) 69 [arXiv:hep-lat/0405027].

[5] D. Galletly et al. [QCDSF Collaboration], PoS LAT2005 (2006) 363 [arXiv:hep-lat/0510050].

[6] R. Frezzotti, P. A. Grassi, S. Sint and P. Weisz [ALPHA collaboration], JHEP 0108 (2001) 058 [arXiv:hep-lat/0101001].

[7] R. Frezzotti and G. C. Rossi, JHEP 0408 (2004) 007 [arXiv:hep-lat/0306014].

[8] K. Jansen, A. Shindler, C. Urbach and I. Wetzorke [XLF Collaboration], Phys. Lett. B 586 (2004) 432 [arXiv:hep-lat/0312013].

[9] K. Jansen, M. Papinutto, A. Shindler, C. Urbach and I. Wetzorke [XLF Collaboration], Phys. Lett. B 619 (2005) 184 [arXiv:hep-lat/0503031]. 
[10] K. Jansen, M. Papinutto, A. Shindler, C. Urbach and I. Wetzorke [XLF Collaboration], JHEP 0509 (2005) 071 [arXiv:hep-lat/0507010].

[11] A. M. Abdel-Rehim, R. Lewis and R. M. Woloshyn, Phys. Rev. D 71 (2005) 094505 [arXiv:hep-lat/0503007].

[12] S. Capitani, K. Jansen, M. Papinutto, A. Shindler, C. Urbach and I. Wetzorke, Phys. Lett. B 639 (2006) 520 [arXiv:hep-lat/0511013].

[13] $\mathrm{Ph}$. Boucaud et al. [ETM Collaboration], arXiv:hep-lat/0701012.

[14] C. Urbach [ETM Collaboration], PoS (LATTICE 2007) 022.

[15] M. Göckeler, R. Horsley, E. M. Ilgenfritz, H. Perlt, P. Rakow, G. Schierholz and A. Schiller, Phys. Rev. D 54 (1996) 5705 [arXiv:hep-lat/9602029].

[16] S. J. Dong and K. F. Liu, Phys. Lett. B 328 (1994) 130 [arXiv:hep-lat/9308015].

[17] M. Foster and C. Michael [UKQCD Collaboration], Phys. Rev. D 59 (1999) 074503 [arXiv:hep-lat/9810021].

[18] C. McNeile and C. Michael [UKQCD Collaboration], Phys. Rev. D 73 (2006) 074506 [arXiv:hep-lat/0603007].

[19] G. Martinelli and C. T. Sachrajda, Phys. Lett. B 196 (1987) 184.

[20] U. Wolff [ALPHA collaboration], Comput. Phys. Commun. 156 (2004) 143 [Erratum-ibid. 176 (2007) 383] [arXiv:hep-lat/0306017].

[21] D. Arndt and M. J. Savage, Nucl. Phys. A 697 (2002) 429 [arXiv:nucl-th/0105045].

[22] J. W. Chen and X. D. Ji, Phys. Lett. B 523 (2001) 107 [arXiv:hep-ph/0105197].

[23] W. Detmold and C. J. Lin, Phys. Rev. D 71 (2005) 054510 [arXiv:hep-lat/0501007]. 